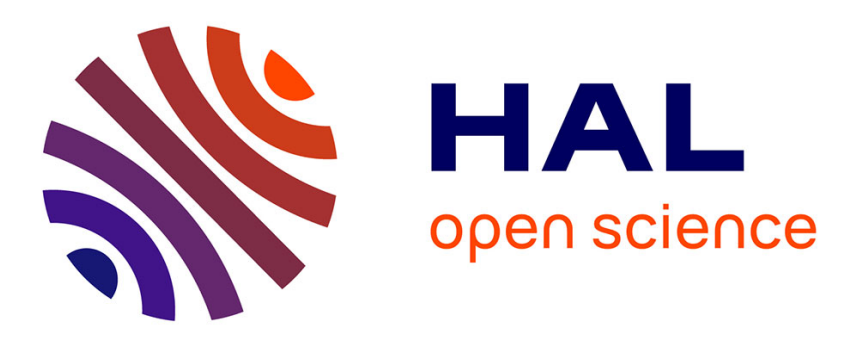

\title{
Collision-induced hyper-Rayleigh spectrum of H2-Ar gas mixture
}

Tadeusz Bancewicz, Waldemar Glaz, Jean-Luc Godet, George Maroulis

\section{To cite this version:}

Tadeusz Bancewicz, Waldemar Glaz, Jean-Luc Godet, George Maroulis. Collision-induced hyperRayleigh spectrum of H2-Ar gas mixture. Journal of Chemical Physics, 2008, 129 (12), pp.124306. 10.1063/1.2981042 . hal-03436307

\section{HAL Id: hal-03436307 https://univ-angers.hal.science/hal-03436307}

Submitted on 19 Nov 2021

HAL is a multi-disciplinary open access archive for the deposit and dissemination of scientific research documents, whether they are published or not. The documents may come from teaching and research institutions in France or abroad, or from public or private research centers.
L'archive ouverte pluridisciplinaire HAL, est destinée au dépôt et à la diffusion de documents scientifiques de niveau recherche, publiés ou non, émanant des établissements d'enseignement et de recherche français ou étrangers, des laboratoires publics ou privés. 


\section{AlP | The Journal of Chemical Physics}

\section{Collision-induced hyper-Rayleigh spectrum of H 2 - Ar gas mixture}

Tadeusz Bancewicz, Waldemar Głaz, Jean-Luc Godet, and George Maroulis

Citation: The Journal of Chemical Physics 129, 124306 (2008); doi: 10.1063/1.2981042

View online: http://dx.doi.org/10.1063/1.2981042

View Table of Contents: http://scitation.aip.org/content/aip/journal/jcp/129/12?ver=pdfcov

Published by the AIP Publishing

\section{Articles you may be interested in}

Asymptotic multipolar expansion of collision-induced properties

J. Chem. Phys. 134, 104309 (2011); 10.1063/1.3562210

Binary rototranslational hyper-Rayleigh spectra of $\mathrm{H} 2-$ He gas mixture

J. Chem. Phys. 131, 204305 (2009); 10.1063/1.3264691

Moments of hyper-Rayleigh spectra of selected rare gas mixtures

J. Chem. Phys. 127, 134308 (2007); 10.1063/1.2772262

The hyper-Rayleigh light scattering spectrum of gaseous $\mathrm{Ne}-\mathrm{Ar}$ mixture

J. Chem. Phys. 118, 6264 (2003); 10.1063/1.1557524

Collision-induced hyper-Rayleigh spectrum of octahedral molecules: The case of SF 6

J. Chem. Phys. 115, 8547 (2001); 10.1063/1.1410979

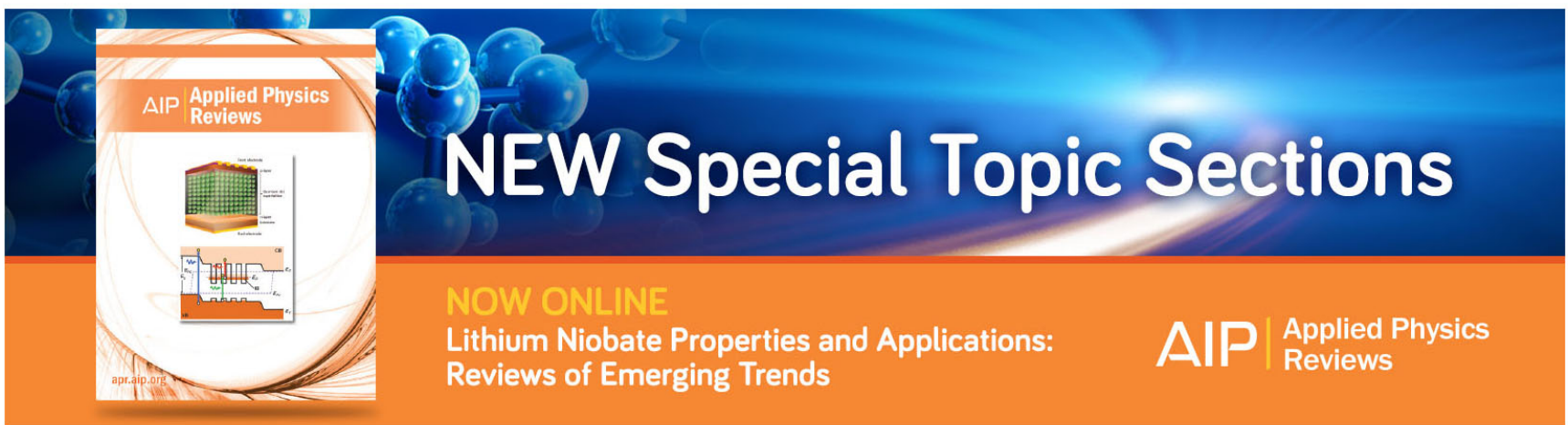




\title{
Collision-induced hyper-Rayleigh spectrum of $\mathbf{H}_{2}-$ Ar gas mixture
}

\author{
Tadeusz Bancewicz,, ${ }^{1, a)}$ Waldemar Głaz, ${ }^{1, b)}$ Jean-Luc Godet, ${ }^{2, c)}$ and George Maroulis ${ }^{3, d)}$ \\ ${ }^{1}$ Nonlinear Optics Division, Faculty of Physics, Adam Mickiewicz University, \\ 61-614 Poznań, Poland \\ ${ }^{2}$ Laboratoire des Propriétés Optiques des Matériaux et Applications, Université d'Angers, \\ 2 Boulevard Lavoisier, 49045 Angers, France \\ ${ }^{3}$ Department of Chemistry, University of Patras, GR-26500 Patras, Greece
}

(Received 14 July 2008; accepted 20 August 2008; published online 24 September 2008)

\begin{abstract}
The collision-induced hyper-Rayleigh (CIHR) spectra of the gaseous $\mathrm{H}_{2}-\mathrm{Ar}$ mixture are discussed in the binary regime on the basis of our ab initio computed $\mathrm{H}_{2}-\mathrm{Ar}$ collision-induced (CI) first dipole hyperpolarizability tensor $\Delta \beta(R)$. A method for the computation of the spherical, rotationally adapted components $\Delta \beta_{\lambda L}^{(s, K)}(R)$ of $\Delta \boldsymbol{\beta}(R)$ needed for spectroscopic line shape analysis is proposed. Both the vector and the septor parts of the $\mathrm{H}_{2}-\mathrm{Ar}$ CIHR spectrum are evaluated at room $(T$ $=295 \mathrm{~K})$ temperature. The spectra are calculated assuming the full quantum computations based on the Schrödinger equation of the relative translational motion of $\mathrm{H}_{2}-\mathrm{Ar}$ as well as semiclassical methods (classical trajectory approach and Birnbaum-Cohen model translational profiles). The $\mathrm{H}_{2}-\mathrm{Ar}$ pair CIHR septor spectrum has been found stronger than the vector one. (C) 2008 American Institute of Physics. [DOI: 10.1063/1.2981042]
\end{abstract}

\section{INTRODUCTION}

The recent rapid progress in experimental methods of molecular spectroscopy as well as in numerical procedures of quantum chemistry $(\mathrm{QC})$ makes it possible to attain a deeper insight into considerably more subtle microscopic molecular phenomena. Among them, the linear collision-induced (CI) Rayleigh/Raman light scattering processes have been treated experimentally and theoretically ${ }^{1-10}$ for decades, both with regard to systems composed of monomers and those consisted of interacting atoms/molecules, forming weakly bound complexes-supermolecules.

Concurrently, nonlinear processes of that kind were also researched into, though on account of relatively weak intensities detected in this case attention was almost exclusively paid to the monomer aspect of the studies. However, the prospect of further development in measuring tools of experimental spectroscopy together with the present day availability of powerful QC codes heralds rapprochement between theory and experiment also in the field of nonlinear CI light scattering. Therefore formulating a theoretical and numerical basis in order to interpret such processes seems to be worth being taken into consideration. ${ }^{11,12}$

In our earlier works ${ }^{13-17}$ we developed numerical/ theoretical methods of evaluating molecular properties active in the CI hypher-Rayleigh (CIHR) events in media of a relatively high (linear) symmetry formed by atomic pairs of unlike noble gas atoms. The present paper succeeds the previ-

\footnotetext{
${ }^{a)}$ Electronic mail: tbancewi@zon12.physd.amu.edu.pl. URL: http:// zon8.physd.amu.edu.pl/ tbancewi

${ }^{b)}$ Electronic mail: glaz@kielich.amu.edu.pl.

${ }^{c)}$ Electronic mail: jean-luc.godet@univ-angers.fr.

${ }^{\mathrm{d})}$ Electronic mail: maroulis@upatras.gr.
}

ous series by extending the scope of discussion to a more complex symmetry of scattering entities, i.e., supermolecular system of $\mathrm{H}_{2}-\mathrm{Ar}$.

The phenomenon of interest to us occurs when at a collision of two molecules the intermolecular interactions lead to distortion of the molecules charge distributions, so that a collisional complex may possess a dipole moment, polarizability, and hyperpolarizability in excess of a sum of these properties of the isolated molecules. ${ }^{3-5}$ Theoretical and experimental investigations of weakly bound molecular complexes are of fundamental importance to our understanding of the intermolecular forces responsible for the properties of dense phases. These interactions may lead in some cases to unusual situations especially when the monomers lack some spectral features that emerge due to intermolecular interactions. These changes are the most prominent if the interacting monomers possess a center of symmetry. Owing to the inversion symmetry, the monomer tensorial electric properties of odd rank vanish. ${ }^{18,19}$ For instance, the permanent dipole moment of such microsystems is strictly zero and as a result no electromagnetic radiation absorption can occur. The radiation is absorbed, however, due to intermolecular interactions. $^{20}$

Similarly, the third rank tensor of the first dipole hyperpolarizability $\boldsymbol{\beta}$ responsible for hyper-Rayleigh (HR) scattering of light is inconsistent with any centrosymmetic microsystem. The third rank tensor is odd under the coordinate inversion, whereas the inversion leaves the centrosymmetic microsystem unchanged. The CI first dipole hyperpolarizability tensor $\Delta \boldsymbol{\beta}(R)$ appears only as a result of the interaction with another microsystem, provided that the pair has configurations which do not possess the center of symmetry. The $\mathrm{H}_{2}$-Ar pair fulfills these conditions.

The CI dipole moment is related to the CI absorption. ${ }^{20,21}$ The trace of the CI polarizability is related to 
the second virial dielectric coefficients $B_{\epsilon},{ }^{22}$ whereas the dynamics of this trace are investigated by analyzing the polarized (isotropic) spectrum of CI Rayleigh light scattering. $7,9,23$ Similarly, the anisotropy of the CI polarizability is related to the depolarized spectrum of CI light scattering ${ }^{10,24-30}$ and/or to the second virial coefficient $B_{K}$ of dc Kerr effect. ${ }^{18,19,27,31}$ The nonlinear effects also contribute to the Kerr effect and dc second harmonic generation. It is noteworthy, however, that in the latter experiments the scalar product of the dipole $(\boldsymbol{\mu})$ and hyperpolarizability $(\boldsymbol{\beta})$ is measured. This means that only the vector part of the hyperpolarizability tensor is accessible by these methods.

CIHR scattering has two components, the vector and the septor ones, separable by polarization effects. ${ }^{19,32-34}$ We note that for the circularly polarized incident laser radiation the septor part of the first dipole hyperpolarizability is directly measurable. $^{32,35,36}$ It is worth noticing that the nonlinear optical (NLO) properties of molecules are of particular importance due to the current interest in NLO materials for highlevel applications in advanced technologies. ${ }^{37,38}$

In this work we report analysis of the CIHR spectrum of the $\mathrm{H}_{2}-\mathrm{Ar}$ mixture at room temperature and in the binary regime. In general, in this spectrum the translational and purely rotational bands form one composite band. The spectroscopic method we proposed here is a powerful tool because we compare the shapes of the spectral distributions on an absolute scale. In Sec. II, the theory and the method of the computation of CI hyperpolarizability for the $\mathrm{H}_{2}-$ Ar pair are presented. The CI pair hyperpolarizability for $D_{\infty h}$ moleculeatom system is considered analytically in Sec. III. The symmetry allowed form of the CI hyperpolarizability spherical harmonic expansion is discussed here as well. The CI spectrum of the HR scattered light for molecular hydrogen-argon system is given in Sec. IV. The quantum and semiclassical (SC) considerations are presented. The potential for $\mathrm{H}_{2}-\mathrm{Ar}$ pair used in our calculations is given in Sec. V. In Sec. VI some details of our numerical computations are presented and discussed. Section VII concludes the paper.

\section{AB INITIO COLLISIONAL HYPERPOLARIZABILITY}

An extensive presentation of our computational methodology for interaction properties may be found in our previous works (see Refs. 39 and 40). A few essential details will be given here. We follow Buckingham's conventions and terminology throughout this section. In the finite-field method the energy of an uncharged molecule in a weak, homogeneous static electric field can be written as

$$
\begin{aligned}
E^{p}= & E^{0}-\mu_{i} F_{i}-\frac{1}{2} \alpha_{i j} F_{i} F_{j}-\frac{1}{6} \beta_{i j k} F_{i} F_{j} F_{k} \\
& -\frac{1}{24} \gamma_{i j k l} F_{i} F_{j} F_{k} F_{l}+\cdots,
\end{aligned}
$$

where $F_{i} \ldots$ is the field, $E^{0}$ is the energy of the free molecule, $\mu_{i}$ is the dipole moment, $\alpha_{i j}$ is the dipole polarizability, $\beta_{i j k}$ is the first dipole hyperpolarizability, and $\gamma_{i j k l}$ is the second dipole hyperpolarizability. The subscripts denote Cartesian components and the repeated subscript implies summation over $x, y$, and $z$. The number of independent and nonzero components needed to specify the above tensors is regulated by symmetry. ${ }^{18,19,41,42}$ The tensorial physical property of a system is characterized by two kinds of symmetry: the intrinsic symmetry defined by the nature of the phenomena studied, described by the symmetry of Cartesian tensor indices and the second one due to the geometric symmetry of the system. We consider the (hyper)polarizability tensors of the supermolecule to be fully symmetric (s) to its indices. The molecules is always on the $z$ axis and the Ar atom moves accordingly. Then for an arbitrary $\mathrm{H}_{2}-\mathrm{Ar}$ configuration $\left(C_{s}\right.$ point group symmetry) the polarizability tensor has four linearly independent components, whereas the hyperpolarizability tensor has six linearly independent components. We compute the CI polarizability and hyperpolarizability tensors for the $\mathrm{H}_{2}-$ Ar pair as a function of their relative separation $R$ and for the orientations of $0^{\circ}, 45^{\circ}$, and $90^{\circ}$ between the relative intermolecular vector $\mathbf{R}$ and the $\mathrm{H}-\mathrm{H}$ bond. In our computations we assumed that $r_{\mathrm{H}-\mathrm{H}}=1.449 a_{0}{ }^{43}$

The interaction electric properties of the supermolecule are obtained via the well-tested Boys-Bernardi counterpoisecorrection method. ${ }^{44}$ The interaction quantity $\mathrm{P}_{\text {int }}\left(\mathrm{H}_{2} \cdots \mathrm{Ar}\right)$ (R) at a given internuclear separation $\mathbf{R}$ is computed as

$$
\begin{aligned}
\mathrm{P}_{\text {int }}\left(\mathrm{H}_{2} \cdots \operatorname{Ar}\right)(\mathbf{R})= & \mathrm{P}\left(\mathrm{H}_{2} \cdots \operatorname{Ar}\right)(\mathbf{R})-\mathrm{P}\left(\mathrm{H}_{2} \cdots X\right)(\mathbf{R}) \\
& -\mathrm{P}(X \cdots \operatorname{Ar})(\mathbf{R}) .
\end{aligned}
$$

All calculations were performed at the second-order MøllerPlesset, MP2(full), level of theory with the GAUSSIAN 98 program. ${ }^{45}$ All electrons were correlated. Full descriptions of the MP2 method are available in standard textbooks. ${ }^{46}$ The selection of suitable basis sets is of central importance to the interaction (hyper)polarizability calculations. In this work we relied on a $[8 s 6 p 5 d 4 f]$ basis set used and tested systematically in the previous works (see Refs. 39 and 40). This basis gives the Ar dipole polarizability at the self-consistent field level of theory: $\alpha / e^{2} a_{0}^{2} E_{\mathrm{h}}^{-1}=10.66$, to be compared to the numerical Hartree-Fock ${ }^{47}$ value of 10.758. For $\mathrm{H}_{2}$ we rely on previous experience ${ }^{48}$ to propose a $[6 s 4 p 2 d]$ basis set. This is an augmented version of the $[6 s 4 p 1 d]$ purposeoriented basis set used previously in the calculations for $\mathrm{H}_{2}-\mathrm{H}_{2}$ (see Ref. 40) which contains an additional diffuse $d$-GTF with exponent $\eta_{d}=0.0464 a_{0}^{-2}$.

\section{CI PAIR HYPERPOLARIZABILITY FOR $D_{\infty}$ MOLECULE-ATOM SYSTEM: SYMMETRY ADAPTED SPHERICAL HARMONIC EXPANSION}

\section{A. The irreducible spherical components of $\Delta \beta(R)$}

For an arbitrary relative geometry the $\mathrm{H}_{2}-$ Ar supermolecule is of $C_{s}$ symmetry. For the T shaped configuration, it reduces to $C_{2 v}$ symmetry, whereas for the L shaped configuration it further reduces to $C_{\infty v}$ symmetry. First we note that the reduction spectrum of a fully index symmetric (s) rank-3 tensor reads: $1 \oplus 3$. Then only the vectorlike (weight- $K=1$ ) and the septorlike (weight- $K=3$ ) parts are present for a tensor like this. This situation applies to the CI hyperpolarizability tensor $\Delta \boldsymbol{\beta}(R)$ considered here. Now we discuss the irreducible spherical components of $\Delta \boldsymbol{\beta}(R)$ for $C_{s}, C_{2 v}$, and $C_{\infty v}$ symmetries. $^{35,49,50}$ For $C_{s}$ symmetry, we have six independent irreducible spherical components, namely, 
TABLE I. The fitting numerical coefficients of the subsequent spherical components of the dipolar $(K=1)$ part of CI hyperpolarizabilty of $\mathrm{H}_{2}-\mathrm{Ar}$ pair. The fitting function is given by Eq. (5).

\begin{tabular}{cccccccc}
\hline \hline$\lambda L$ & $a$ & $b$ & $c$ & $d$ & $e$ & $f$ & $g$ \\
\hline 01 & -6.317 & -22.992 & 10.619 & -0.0134 & $4.924 \times 10^{-5}$ & $5.350 \times 10^{-5}$ & $\cdots$ \\
21 & 3.431 & -6.578 & 2.527 & 0.0057 & -0.0013 & $4.174 \times 10^{-5}$ & $\cdots$ \\
23 & $5.006 \times 10^{-10}$ & -26.232 & 45.763 & -0.0088 & 0.0014 & $-3.115 \times 10^{-5}$ & -0.13356 \\
45 & -18.390 & -5.132 & -4.354 & 0.006 & -0.0005 & $1.081 \times 10^{-5}$ & $\cdots$ \\
\hline \hline
\end{tabular}

(a) two for the vector part: $\Delta \beta_{0}^{(s, 1)}(R)$ and $\Delta \beta_{1}^{(s, 1)}(R)$ $=\Delta \beta_{-1}^{(s, 1)}(R)$ and

(b) four for the septor part: $\Delta \beta_{0}^{(s, 3)}(R), \quad \Delta \beta^{(s, 3)}(R)$ $=\Delta \beta_{-1}^{(s, 3)}(R), \quad \Delta \beta_{2}^{(s, 3)}(R)=\Delta \beta_{-2}^{(s, 3)}(R), \quad$ and $\quad \Delta \beta_{3}^{(s, 3)}(R)$ $=\Delta \beta_{-3}^{(s, 3)}(R)$.

The relations between the $a b$ initio computed Cartesian components (see Sec. II) and the irreducible spherical CI hyperpolarizability ones are given in Ref. 51 . We note that the set of irreducible spherical components $\Delta \beta_{\mu}^{(s, K)}(R)$ reduces to three $\Delta \beta_{0}^{(s, 1)}(R), \Delta \beta_{0}^{(s, 3)}(R)$, and $\Delta \beta_{ \pm 2}^{(s, 3)}(R)$ independent components for $C_{2 v}$ symmetry (for the T shaped geometry) and, finally, to the two $\Delta \beta_{0}^{(s, 1)}(R)$ and $\Delta \beta_{0}^{(s, 3)}(R)$ independent components for $C_{\infty \infty V}$ symmetry (for the L shaped geometry).

\section{B. Symmetry adapted form of the hyperpolarizability spherical harmonic expansion}

The CI pair hyperpolarizability tensor $\Delta \boldsymbol{\beta}$ for a linear molecule-atom supermolecule is dependent on the molecular orientation $\Omega$ and the relative intermolecular separation vector $\mathbf{R}$. We expand $\Delta \beta_{\mu}^{(s, K)}(R)$ in terms of spherical harmonics ${ }^{20,52,53}$

$$
\begin{aligned}
\Delta \beta_{\mu}^{(s, K)}(R)= & \frac{4 \pi}{(2 K+1)^{1 / 2}} \sum_{\lambda L} \Delta \beta_{\lambda L}^{(s, K)}(R)\left\{\mathbf{Y}_{\lambda}(\Omega)\right. \\
& \left.\otimes \mathbf{Y}_{L}(\hat{\mathbf{R}})\right\}_{\mu}^{K}
\end{aligned}
$$

where $\Delta \beta_{\lambda L}^{(s, K)}(R)$ stands for the symmetry adapted components of $\Delta \boldsymbol{\beta}(R)$. We choose the intermolecular separation vector $\hat{\mathbf{R}}$ to be parallel to the $z$-axis; hence, $Y_{L M}=\sqrt{(2 L+1) / 4 \pi} \delta_{M 0}$ and Eq. (3) transforms into

$$
\begin{aligned}
\Delta \beta_{\mu}^{(s, K)}(R)= & \left(\frac{4 \pi}{2 K+1}\right)^{1 / 2} \sum_{\lambda L}(2 L+1)^{1 / 2} \\
& \times \Delta \beta_{\lambda L}^{(s, K)}(R) Y_{\lambda \mu}(\Omega) C_{\lambda \mu L 0}^{K \mu},
\end{aligned}
$$

where $C_{a \alpha b \beta}^{c \gamma}$ stands for the Clebsch-Gordan coefficient. ${ }^{54}$ For the totally symmetric hyperpolarizability tensor $\Delta \boldsymbol{\beta}(R)$, $K$ must be equal to 1 and/or 3. For homonuclear molecules $\left(\mathrm{H}_{2}\right.$ in the $\mathrm{H}_{2}-$ Ar pair) $\lambda$ of Eq. (4) must be an even integer whereas $\lambda+L$ must be odd. Moreover, $|K-\lambda| \leq L \leq K+\lambda$. The system of equations given by relation (4) have been solved $^{55}$ for the rotationally adapted components $\Delta \beta_{\lambda L}^{(s, K)}(R)$ using the known $\Delta \beta_{\mu}^{(s, K)}(R)$ irreducible spherical components computed for selected $\mathrm{H}_{2}-\mathrm{Ar}$ configurations and selected $\mathrm{H}_{2}-\mathrm{Ar}$ distances. Using the ab initio computed Cartesian components of the CI hyperpolarizability tensor for the dipolar (or vector; $K=1$ ) part of $\Delta \boldsymbol{\beta}(R)$, we are able to compute four spherical rotationally adapted components, namely, $\Delta \beta_{01}^{(s, 1)}(R), \Delta \beta_{21}^{(s, 1)}(R), \Delta \beta_{23}^{(s, 1)}(R)$, and $\Delta \beta_{45}^{(s, 1)}(R)$. Similarly, for the octopolar (or septor; $K=3$ ) part, the set of equations given by relation (4) has been solved for seven octopolar irreducible rotationally adapted components $\Delta \beta_{\lambda L}^{(s, 3)}(R)$, namely, $\quad \Delta \beta_{03}^{(s, 3)}(R), \quad \Delta \beta_{21}^{(s, 3)}(R), \quad \Delta \beta_{23}^{(s, 3)}(R), \quad \Delta \beta_{25}^{(s, 3)}(R)$, $\Delta \beta_{41}^{(s, 3)}(R), \Delta \beta_{43}^{(s, 3)}(R)$, and $\Delta \beta_{45}^{(s, 3)}(R)$ from their known Cartesian ones. The numerical results have been presented elsewhere. ${ }^{51,55,56}$ The fitting analytical forms are also available. We fit the numerical data with the following function:

$$
\begin{aligned}
\Delta \beta_{\lambda L}^{(s, K)}(R)= & a \exp \left[b(R / 10)^{2}+c(R / 10)\right]+\frac{g}{(R / 10)^{4}} \\
& +\frac{d}{(R / 10)^{7}}+\frac{e}{(R / 10)^{9}}+\frac{f}{(R / 10)^{11}} .
\end{aligned}
$$

The fitting numerical coefficients of the vector part of the first dipole ${ }^{3}$ hyperpolarizability of the $\mathrm{H}_{2}-\mathrm{Ar}$ pair are

TABLE II. The fitting numerical coefficients of the subsequent spherical components of the octopolar $(K=3)$ part of CI hyperpolarizabilty of $\mathrm{H}_{2}-$ Ar pair. The fitting function is given by Eq. (5).

\begin{tabular}{rcrccccr}
\hline \hline$\lambda L$ & $a$ & $b$ & $c$ & $d$ & $e$ & $f$ & \multicolumn{1}{c}{$g$} \\
\hline 03 & -3.95 & -1.566 & 0.183 & -0.025 & -0.0014 & $5.999 \times 10^{-5}$ & \multicolumn{1}{c}{0.81} \\
21 & 4.80 & 0.502 & -3.577 & 0.0049 & $3.14 \times 10^{-4}$ & $-1.339 \times 10^{-5}$ & -0.190 \\
23 & -3.84 & 0.327 & -3.047 & -0.01 & $3.216 \times 10^{-4}$ & $-1.909 \times 10^{-7}$ & 0.255 \\
25 & 0.09 & -1.964 & 2.882 & 0.025 & -0.0013 & $1.072 \times 10^{-5}$ & -0.293 \\
41 & $2.437 \times 10^{-6}$ & -5.732 & 15.364 & 0.0048 & $-3.823 \times 10^{-4}$ & $8.381 \times 10^{-6}$ & -0.031 \\
43 & -0.24 & -1.587 & -1.067 & -0.0049 & $4.103 \times 10^{-4}$ & $-9.045 \times 10^{-6}$ & 0.036 \\
45 & 1.26 & 0.816 & -4.266 & 0.0045 & $-3.697 \times 10^{-4}$ & $7.703 \times 10^{-6}$ & -0.036 \\
\hline \hline
\end{tabular}


collected in Table I. The fitting numerical coefficients of the septor part are collected in Table II. All data there are given in a.u.

\section{CI SPECTRUM OF HR SCATTERED LIGHT FOR MOLECULAR HYDROGEN-ARGON SYSTEM}

\section{A. General considerations}

We consider the right angle geometry for observation of the CIHR spectra with no analyzer in the experimental setup. We assume the incident laser beam of frequency $\omega$ to be polarized in the scattering plane. For these conditions we observe the depolarized $\left(I_{H}\right)$ CIHR light scattering spectra. By assuming of separability of rotational and translational motions the double differential cross section for the CIHR depolarized component reads ${ }^{16}$

$$
\begin{aligned}
\left(\frac{\partial^{2} I_{H}^{2 \omega_{L}}}{\partial \Omega \partial \omega}\right) / I_{0}^{2}= & \frac{\pi}{2 c} k_{s}^{4}\left\{\frac{2}{45} \sum_{\lambda L} \sum_{j j^{\prime}} P_{j}(2 j+1)\left(2 j^{\prime}+1\right)\right. \\
& \times\left(\begin{array}{ccc}
j & \lambda & j^{\prime} \\
0 & 0 & 0
\end{array}\right)^{2} g_{\lambda L}^{(1)}\left(\omega-\omega_{j j^{\prime}}\right) \\
& +\frac{8}{105} \sum_{\lambda L} \sum_{j j^{\prime}} P_{j}(2 j+1)\left(2 j^{\prime}+1\right) \\
& \left.\times\left(\begin{array}{ccc}
j & \lambda & j^{\prime} \\
0 & 0 & 0
\end{array}\right)^{2} g_{\lambda L}^{(3)}\left(\omega-\omega_{j j^{\prime}}\right)\right\},
\end{aligned}
$$

where $\omega=2 \pi \nu$ stands for the angular frequency shift. Considering the incident laser beam to be polarized perpendicularly to the scattering plane we obtain the polarized $\left(I_{V}\right)$ component of the CIHR spectra

$$
\begin{aligned}
\left(\frac{\partial^{2} I_{V}^{2 \omega_{L}}}{\partial \Omega \partial \omega}\right) / I_{0}^{2}= & \frac{\pi}{2 c} k_{s}^{4}\left\{\frac{2}{9} \sum_{\lambda L} \sum_{j j^{\prime}} P_{j}(2 j+1)\left(2 j^{\prime}+1\right)\right. \\
& \times\left(\begin{array}{ccc}
j & \lambda & j^{\prime} \\
0 & 0 & 0
\end{array}\right)^{2} g_{\lambda L}^{(1)}\left(\omega-\omega_{j j^{\prime}}\right) \\
& +\frac{2}{21} \sum_{\lambda L} \sum_{j j^{\prime}} P_{j}(2 j+1)\left(2 j^{\prime}+1\right) \\
& \left.\times\left(\begin{array}{ccc}
j & \lambda & j^{\prime} \\
0 & 0 & 0
\end{array}\right)^{2} g_{\lambda L}^{(3)}\left(\omega-\omega_{j j^{\prime}}\right)\right\} .
\end{aligned}
$$

In Eqs. (6) and (7),

$$
\left(\begin{array}{lll}
J & \lambda & J^{\prime} \\
0 & 0 & 0
\end{array}\right)
$$

denotes the $3-j$ Wigner symbol, and $P_{j}$ is the Boltzmann population factor. The $g_{\lambda L}^{(K)}(\omega)$ functions appearing in Eqs. (6) and (7) represent the translational profiles stemming from the successive symmetry adapted components $\Delta \beta_{\lambda L}^{(s, K)}(R)$ of, respectively, the vector and the septor parts of the CI hyperpolarizability tensor. We note that each translational function is shifted by a frequency of the hydrogen molecule rotational transition $\omega_{j j^{\prime}}$. We use

$$
P_{j}=\frac{g_{j} \exp \left(-E_{j} / k_{B} T\right)}{\sum_{j} g_{j}(2 j+1) \exp \left(-E_{j} / k_{B} T\right)}
$$

for the Boltzmann population factor and

$$
\omega_{j j^{\prime}}=-B\left[j^{\prime}\left(j^{\prime}+1\right)-j(j+1)\right],
$$

where $g_{j}$ stands for the nuclear-statistical weight factor (for hydrogen $g_{j}=1$ if $j$ is even and $g_{j}=3$ if $j$ is odd), whereas $B$ stands for the rotational constant of the hydrogen molecule.

We assume that the $\mathrm{H}_{2}-\mathrm{Ar}$ intermolecular potential is spherical (or almost spherical). That assumption splits the autocorrelation function $F(t)$ of the CIHR scattered radiation into the product ( $V$ is the active scattering volume),

$$
F(t)=\frac{1}{V} r^{\mathrm{rot}}(t) g^{\mathrm{tr}}(t)
$$

of the rotational $r^{\mathrm{rot}}(t)$ and the translational $g^{\mathrm{tr}}(t)$ components and, consequently, the spectral distribution (10) takes the form of the convolution

$$
\frac{\partial^{2} \sigma}{\partial \Omega \partial \omega}=k_{s}^{4} \int\left(\frac{\partial^{2} \sigma}{\partial \Omega \partial \omega^{\prime}}\right)^{\operatorname{rot}}\left(\frac{\partial^{2} \sigma}{\partial \Omega \partial\left(\omega-\omega^{\prime}\right)}\right)^{\operatorname{tr}} d \omega^{\prime} .
$$

Using Eq. (3) for the translational component, we write

$$
\begin{aligned}
g_{\lambda L}^{(K)}(t)= & \frac{4 \pi}{2 L+1}\left\langle\Delta \beta_{\lambda L}^{(s, K)}(R(0)) \mathbf{Y}_{L}(\hat{\mathbf{R}}(0)) \odot\right. \\
& \left.\times \Delta \beta_{\lambda L}^{(s, K)}(R(t)) \mathbf{Y}_{L}(\hat{\mathbf{R}}(t))\right\rangle .
\end{aligned}
$$

Then for $I_{\mathrm{H}}$ scattering geometry, the double differential cross section of the vector part of the CIHR scattered radiation reads

$$
\begin{aligned}
\left(\frac{\partial^{2} I_{H}^{2 \omega_{L}}}{\partial \Omega \partial \omega}\right)_{\text {vector }} / I_{0}^{2}= & \frac{2}{45} \frac{\pi}{2 c} k_{s}^{4} \sum_{\lambda, L} \sum_{j, j^{\prime}}(2 j+1) \\
& \times\left(2 j^{\prime}+1\right) P_{j}\left(\begin{array}{ccc}
j & \lambda & j^{\prime} \\
0 & 0 & 0
\end{array}\right)^{2} \\
& \times g_{\lambda L}^{(1)}\left(\omega-\Delta \omega_{j j^{\prime}}\right) .
\end{aligned}
$$

Considering the same scattering conditions, for the septor part of the CIHR spectra we obtain

$$
\begin{aligned}
\left(\frac{\partial^{2} I_{\mathrm{H}}^{2 \omega_{L}}}{\partial \Omega \partial \omega}\right)_{\text {septor }} / I_{0}^{2}= & \frac{8}{105} \frac{\pi}{2 c} k_{s}^{4} \sum_{\lambda, L} \sum_{j, j^{\prime}}(2 j+1) \\
& \times\left(2 j^{\prime}+1\right) P_{j}\left(\begin{array}{ccc}
j & \lambda & j^{\prime} \\
0 & 0 & 0
\end{array}\right)^{2} \\
& \times g_{\lambda L}^{(3)}\left(\omega-\Delta \omega_{j j^{\prime}}\right) .
\end{aligned}
$$

The rotational stick spectra associated to the vector part and the septor parts of the CIHR spectra being defined by Eqs. (13) and (14), it remains to compute the translational parts $g_{\lambda L}^{(1)}(\omega)$ and $g_{\lambda L}^{(3)}(\omega)$. We note that the translation correlation function $g_{\lambda L}^{(K)}(\omega)$ is the Fourier transform of the timedependent correlation function $g_{\lambda L}^{(K)}(t)$ given by Eq. (12). In this work, we present the corresponding quantum mechanical (QM) calculations and two SC methods as well. 
TABLE III. The BC SC parameters used in our calculations. The values of $\tau_{1}$ and $\tau_{2}$ are in $10^{-14} \mathrm{~s}$. Those of $M_{0}^{*}$ are in $10^{-80} \mathrm{~cm}^{12} \mathrm{erg}^{-1}$.

\begin{tabular}{ccccccc}
\hline \hline$\lambda L$ & ${ }^{(1 \lambda L)} \tau_{1}$ & ${ }^{(1 \lambda L)} \tau_{2}$ & ${ }^{(3 \lambda L)} \tau_{1}$ & ${ }^{(3 \lambda L)} \tau_{2}$ & ${ }^{(1 \lambda L)} M_{0}^{*}$ & ${ }^{(3 \lambda L)} M_{0}^{*}$ \\
\hline 01 & 2.973 & 3.432 & $\ldots$ & $\ldots$ & $1.394 \times 10^{-6}$ & $\ldots$ \\
03 & $\ldots$ & $\ldots$ & 2.728 & 6.391 & $\ldots$ & $5.029 \times 10^{-6}$ \\
21 & 5.283 & 9.42 & 4.201 & 5.075 & $2.004 \times 10^{-6}$ & $2.866 \times 10^{-7}$ \\
23 & 5.001 & 5.441 & 4.133 & 5.553 & $9.979 \times 10^{-6}$ & $6.464 \times 10^{-7}$ \\
25 & $\ldots$ & $\ldots$ & 3.092 & 10.81 & $\ldots$ & $1.321 \times 10^{-6}$ \\
41 & $\ldots$ & $\ldots$ & 11.43 & 8.578 & $\ldots$ & $6.148 \times 10^{-8}$ \\
43 & $\ldots$ & $\ldots$ & 12.06 & 5.148 & $\ldots$ & $7.044 \times 10^{-9}$ \\
45 & 3.715 & 4.639 & 13.57 & 5.085 & $1.016 \times 10^{-9}$ & $9.428 \times 10^{-9}$ \\
\hline \hline
\end{tabular}

\section{B. QM spectrum}

The basic quantity to be counted when dealing with the radial part of the CIHR light scattering spectrum is the matrix element of the radial-dependent rotationally adapted spherical component $\Delta \beta_{\lambda L}^{(s, K)}(R)$,

$$
\begin{aligned}
{ }^{(\mathrm{QM})} g_{\lambda L}^{(K)}(\omega)= & \sum_{i, i^{\prime}}(2 l+1) H(L)_{l}^{l^{\prime}} P_{i} \int_{0}^{\infty} \mid \Psi\left(R ; E^{\prime}, l^{\prime}\right)^{*} \\
& \times\left.\Delta \beta_{\lambda L}^{(s, K)}(R) \Psi(R ; E, l) d R\right|^{2} \delta\left(\omega-\omega_{i^{\prime} i}\right),
\end{aligned}
$$

where

$$
H(L)_{l}^{l^{\prime}}=\left(2 l^{\prime}+1\right)\left(\begin{array}{lll}
l^{\prime} & L & l \\
0 & 0 & 0
\end{array}\right)^{2}
$$

The radial wave functions $\Psi(R ; E, l) / R$ are solutions of the Schrödinger equation of relative translational motion of $\mathrm{H}_{2}$ and $\mathrm{Ar}^{20}$ Then, following the usual path of calculation of such integrals, neglecting the contribution of the bound dimers, we arrive at

$$
\begin{aligned}
(\mathrm{QM}) g_{\lambda L}^{(K)}(\omega)= & \frac{L_{0}^{3}}{V}\left(\frac{2 \mu}{\hbar \pi^{2}}\right) \sum_{l, \Delta l}(2 l+1) H(L)_{l}^{l^{\prime}} \int_{0}^{\infty} \frac{d E}{\left(E E^{\prime}\right)^{1 / 2}} \\
& \times P_{E}\left|\left(\Delta \beta_{\lambda L}^{(s, K)}\right)_{l}^{l^{\prime}}(E, \omega)\right|^{2},
\end{aligned}
$$

where $\mu$ is the reduced mass of the supermolecule and $L_{0}$ is the thermal de Broglie wavelength of the relative motion of two atoms. Moreover,

$$
\begin{aligned}
\left|\left(\Delta \beta_{\lambda L}^{(s, K)}\right)_{l}^{l^{\prime}}(E, \omega)\right|^{2}= & \mid \int_{0}^{\infty} \Psi^{*}\left(R ; E^{\prime}, l^{\prime}\right) \\
& \times\left.\Delta \beta_{\lambda L}^{(s, K)}(R) \Psi(R ; E, l) d R\right|^{2}, \\
& E^{\prime}=E+\hbar \omega .
\end{aligned}
$$

After changing the normalization conditions, Eq. (17) finally leads to

$$
{ }^{(\mathrm{QM})} g_{\lambda L}^{(K)}(\omega)=\hbar \frac{L_{0}^{3}}{V} \sum_{l, \Delta l}(2 l+1) H(L)_{l}^{l^{\prime}}\left(\Delta \widetilde{\mathcal{B}}_{\lambda L}^{(s, K)}\right)_{l}^{l^{\prime}}(\omega),
$$

where

$$
\left(\Delta \widetilde{\mathcal{B}}_{\lambda L}^{(s, K)}\right)_{l}^{l^{\prime}}(\omega)=\int_{0}^{\infty} d E \exp \left[-E / k_{B} T\right]\left|\left(\Delta \beta_{\lambda L}^{(s, K)}\right)_{l}^{l^{\prime}}(E, \omega)\right|^{2}
$$

Much of the details of the quantum numerical method is given in Refs. 27, 57, and 58 and in the papers cited there. The routine is based on the Numerov algorithm of solving differential equations together with standard integration procedures applied to evaluate necessary matrix elements.

\section{SC spectrum}

\section{Birnbaum-Cohen model spectrum}

For the computation of $g_{\lambda L}^{(1)}(\omega)$ and $g_{\lambda L}^{(3)}(\omega)$, the translational parts of the SC spectra, two methods can be alternately applied. The first one is the Birnbaum-Cohen (BC) model. ${ }^{59}$ By using the sum rules, ${ }^{17}$ we can compute $M_{0}, M_{2}$, and $M_{4}$, the zeroth, second, and fourth classical moments of a given translational spectrum, and deduce the characteristic times $\tau_{1}=\left(M_{0} / M_{2}\right)^{1 / 2} \times y$ and $\tau_{2}=\left(M_{0} / M_{2}\right)^{1 / 2} / y$, where $y=\left(M_{4} M_{0} /\left(3 M_{2}^{2}\right)-1\right)^{1 / 2}$. It is also possible to use $M_{0}^{*}, M_{1}^{*}$, and $M_{2}^{*}$, the SC moments, ${ }^{17}$ on the condition that $\tau_{1}=\left(M_{0}^{*} / M_{1}^{*}\right) \times y^{*}$ and $\tau_{2}=\tau_{0} / y^{*}$, where $\tau_{0}=\hbar /\left(2 k_{B} T\right)$ and $y^{*}=\left(\tau_{0}\left(M_{2}^{*} / M_{1}^{*}-M_{1}^{*} / M_{0}^{*}\right)-1\right)^{1 / 2}$. For the expansion parameters $K, \lambda$, and $L$, the $\mathrm{BC}$ translational part reads

$$
\begin{aligned}
{ }^{(\mathrm{BC})} g_{\lambda L}^{(K)}(\omega)= & \frac{{ }^{(K \lambda L)} M_{0}^{(*)(K \lambda L)} \tau_{1}}{\pi} \exp \left[{ }^{(K \lambda L)} \tau_{2} /{ }^{(K \lambda L)} \tau_{1}\right] \\
& \times \exp \left(\omega \tau_{0}\right) \frac{{ }^{(K \lambda L)} z K_{1}\left({ }^{(K \lambda L)} z\right)}{1+\left(\omega^{(K \lambda L)} \tau_{1}\right)^{2}}
\end{aligned}
$$

where ${ }^{(K \lambda L)} z=\left[\left(1+\left(\omega^{(K \lambda L)} \tau_{1}\right)^{2}\left({ }^{(K \lambda L)} \tau_{2}^{2}+\tau_{0}^{2}\right)\right]^{1 / 2} /{ }^{(K \lambda L)} \tau_{1}\right.$. The parameters of the $\mathrm{BC}$ profiles used here are $\mathrm{SC}$ and given in Table III. Indeed, in the $\mathrm{H}_{2}-\mathrm{Ar}$ case, the differences between the classical times and the SC ones are relatively small.

\section{Classical trajectories spectrum}

The calculation of the translational parts of the SC spectra can be carried out according to a standard procedure which is based on the calculation of the classical trajectories of interacting molecules. ${ }^{27,60}$ We have previously presented the particular aspects of the computation for the dipolar (vector) and octopolar (septor) contributions to the HR classical spectra. ${ }^{15}$ However, the obtained spectral profiles are classical and therefore symmetric. To get ${ }^{(\mathrm{SC})} g_{\lambda L}^{(K)}(\omega)$, the corre- 
sponding SC intensities, the classical translational profiles ${ }^{(\mathrm{CL})} g_{\lambda L}^{(K)}(\omega)$ must be multiplied by an appropriate desymmetrization function $\Lambda(\omega)$, according to the detailed balance principle. The most common desymmetrization functions are $\Lambda_{0}(\omega)=\exp \left(\omega \tau_{0}\right) / \cosh \left(\omega \tau_{0}\right)$, $\Lambda_{1}(\omega)=\omega \tau_{0} \exp \left(\omega \tau_{0}\right) / \sinh \left(\omega \tau_{0}\right), \quad$ and $\quad \Lambda_{2}(\omega)=\exp \left(\omega \tau_{0}\right){ }^{20}$ Because $\Lambda_{0}(\omega)<\Lambda_{1}(\omega)<\Lambda_{2}(\omega)$ for $\omega \neq 0$, it is possible to set $\Lambda(\omega)=\Sigma_{i} a_{i} \Lambda_{i}(\omega)$, where $\Sigma_{i} a_{i}=1$, so as to recover as closely as possible the SC moments $M_{0}^{*}, M_{1}^{*}$, and $M_{2}^{*}$ obtained by the sum rules ${ }^{17}$ by integrating the SC intensities. In that case, the values of the coefficients $a_{i}$ must be adjusted so that the following equation can be approximately verified for $n=0,1$, and 2 ,

$$
{ }^{(K \lambda L)} M_{n}^{*}=\frac{1}{\pi^{2}}\left(\frac{\lambda_{L} / 2}{2 \pi}\right)^{4} \int_{-\infty}^{+\infty} \Lambda(\omega)^{(\mathrm{CL})} g_{\lambda L}^{(K)}(\omega) \omega^{n} d \omega,
$$

where $\lambda_{L}$ is the laser wavelength. The classical intensities obtained by computing molecular trajectories have been desymmetrized by using linear combinations of $\Lambda_{0}(\omega)$ and $\Lambda_{2}(\omega)$ in order to recover the sum rules values of $M_{0}^{*}$ and $M_{2}^{*}$ as closely as possible. The obtained desymmetrization functions $\Lambda(\omega)$ are close together irrespective of $K, \lambda$, and $L$. Moreover, they are close to $\Lambda_{1}(\omega)$ at low frequency shifts $\left(\nu<600 \mathrm{~cm}^{-1}\right)$ and lay between $\Lambda_{1}(\omega)$ and $\Lambda_{2}(\omega)$ at high frequencies. The differences between the corresponding SC translational profiles and the QM computed ones are generally hardly visible on a logarithmic scale. For example, for the translational component $K \lambda L=101$, the root mean square of the relative differences is equal to $5.5 \%$ between $\nu=0$ and $1500 \mathrm{~cm}^{-1}$. The discrepancies between the SC or QM translational profiles and these of the $\mathrm{BC}$ model are generally more pronounced, in particular for $\lambda=4$ for which the computed QM and SC translational profiles are less regular and less close to the BC profile than for $\lambda=0$ or $\lambda=2$. However, the convolution process reduced the differences. As far as their respective translational profiles are convoluted to the same rotational stick spectrum, the three methods of calculation $\mathrm{QM}, \mathrm{SC}$, and $\mathrm{BC}$ finally provide total spectra which are very close on a logarithmic scale. Considering the spectra $I_{V}$ and $I_{H}$ above $\nu=-1000 \mathrm{~cm}^{-1}$ and below $+1800 \mathrm{~cm}^{-1}$, the root mean squares of the relative differences between the QM and SC intensities taken relatively to the QM intensities are equal to $2.8 \%$ and $3.9 \%$, respectively. These root mean squares become $6.0 \%$ and $5.8 \%$ for the relative differences between the QM and BC intensities. By taking into account the possible numerical discrepancies, on one hand, the likely experimental errors, on the other hand, these differences are not really significant in the aforementioned frequency range. For the $\mathrm{H}_{2}-\mathrm{Ar}$ system at room temperature, the $\mathrm{QM}, \mathrm{BC}$, and SC calculations might be equally used.

\section{POTENTIAL}

In our computations we tested the isotropic part of the so-called BC potential of LeRoy et al. ${ }^{61}$ We also considered the symmetry adapted potential of Williams et al. ${ }^{62}$ We noted that the isotropic part of that potential coincides strongly

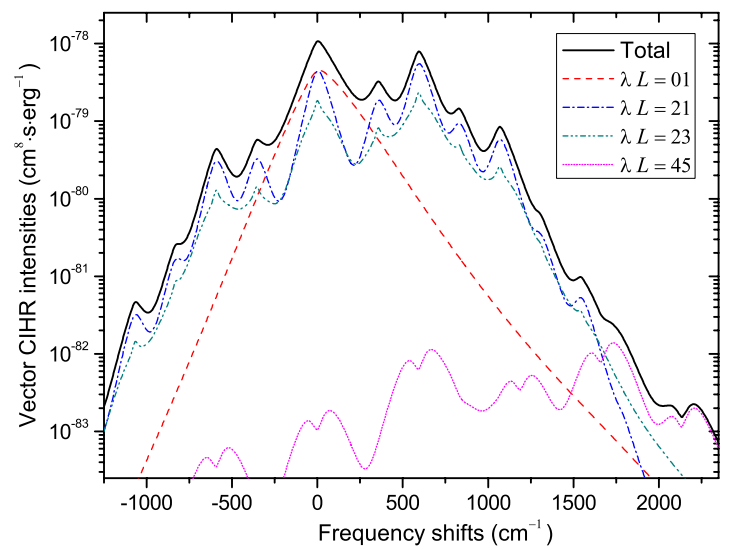

FIG. 1. (Color online) Dipolar (vector) part of the HR spectrum of $\mathrm{H}_{2}-\mathrm{Ar}$. The CIHR intensity (in absolute units: $\mathrm{cm}^{8} \mathrm{~s} / \mathrm{erg}$ ) is computed vs wavenumber (in $\mathrm{cm}^{-1}$ ) for the wavelength of the yttrium aluminum garnet (YAG) laser $\lambda_{L}=1064 \mathrm{~nm}$ by using a $\mathrm{SC}$ calculation (trajectories). From top to bottom at zero frequency: total intensity (solid line), 01-purely translational contribution (dashed line), 21-contribution (dashed-dotted line), 23contribution (dash-dot-dot), and 45-contribution (dotted line).

with the isotropic part of the potential of Refs. 61 and 63. Eventually, in our computations, we decided to use the data of $\mathrm{BC}$ potential of Ref. 61.

\section{RESULTS AND DISCUSSION}

The work presents a comprehensive numerical and theoretical study on NLO CI properties of molecular system of symmetry $C_{s}$ and on related radiative phenomena, namely, the CIHR light scattering effects. The results obtained are partially illustrated by the figures included.

The profiles of the CIHR $\mathrm{H}_{2}-\mathrm{Ar}$ spectrum attributed to irreducible rotationally adapted components $\Delta \beta_{\lambda L}^{(s, 1)}(R)$ $(\lambda L=01,21,23$, and 45) of the vector part of $\Delta \boldsymbol{\beta}(R)$, given in absolute units, are presented in Fig. 1. These graphs enable an assessment of the relative contributions of the particular components in shaping the total vector spectrum. We note that the very low value of $\lambda L=45$ relative to other ones indicates a satisfactory convergence of the series given by Eq. (4) used to compute the $\Delta \beta_{\lambda L}^{(s, 1)}(R)$, even for a relatively small number of the terms applied. As a final result, Fig. 1 shows also the total vector CIHR spectrum.

In turn, Fig. 2 presents the CIHR $\mathrm{H}_{2}-\mathrm{Ar}$ profiles for different irreducible rotationally adapted components of the septor part of the hyperpolarizability tensor, $\Delta \beta_{\lambda L}^{(s, 3)}(R)$, together with the resulting spectral line. In this figure we plotted the pure translational spectrum associated with the $\Delta \beta_{03}^{(s, 3)}(R)$ component and then the resulting spectrum given by $\lambda=2$ contributions, namely, $\Delta \beta_{2 L}^{(s, 3)}(R),(L=1,3,5)$. Additionally, we also drew the global spectrum associated with $\lambda=4: \Delta \beta_{4 L}^{(3)}(R),(L=1,3,5)$. Again, the very low value of the CIHR intensity assigned to $4 L$ components in relation to the other parts indicates a satisfactory convergence of the series given by Eq. (4), even for a relatively small number of the terms used. We note however, that for frequencies above $1600 \mathrm{~cm}^{-1}$, the rotational transitions $\Delta \lambda=4$, explicitly resulting from the $\Delta \beta_{4 L}^{(s, 1)}(R)$ hyperpolarizability components have prominent influence on the CIHR spectrum. 


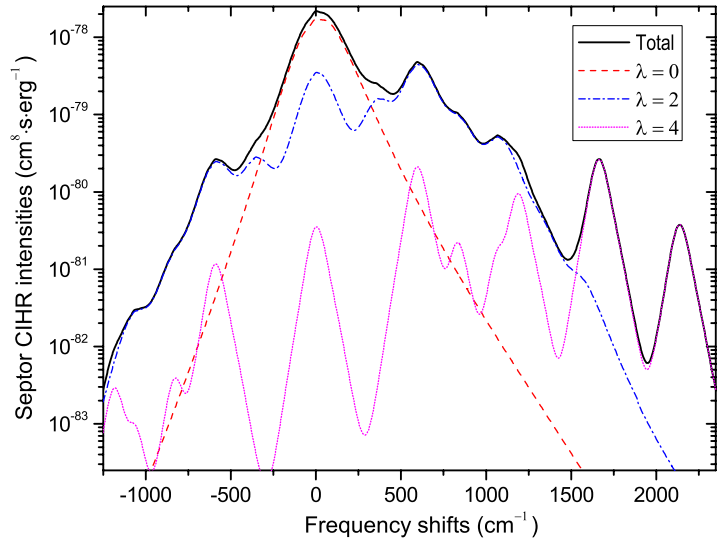

FIG. 2. (Color online) Octopolar (septor) part of the HR spectrum of $\mathrm{H}_{2}-\mathrm{Ar}$. The CIHR intensity (in absolute units: $\mathrm{cm}^{8} \mathrm{~s} / \mathrm{erg}$ ) is computed vs wavenumber $\left(\right.$ in $\mathrm{cm}^{-1}$ ) for the wavelength of the YAG laser $\lambda_{L}=1064 \mathrm{~nm}$ by using a QM calculation. From top to bottom at zero frequency: total intensity (solid line), 03-purely translational contribution (dashed line), $\lambda$ $=2$ contributions (dashed-dotted line), and $\lambda=4$ contributions (dotted line).

In the dc Kerr effect and the dc second harmonic generation experiments, only the projection of the $\boldsymbol{\beta}$ tensor along the permanent dipole moment is accessible. Using the dc second harmonic generation experiment, Donley and Shelton ${ }^{64}$ studied the vector parts of the second CI hyperpolarizability for atom gas mixtures. One of the advantages of HR spectroscopy is the possibility of studying the vector and the septor part of the first hyperpolarizability tensor separately by considering the polarization effects. For the CIHR light scattering conditions assumed here for the septor CIHR light scattering spectrum, we obtain

$$
\left(\frac{\partial^{2} I_{H}^{2 \omega_{L}}}{\partial \Omega \partial \omega}\right)_{\text {septor }}=\frac{7}{2}\left(5\left(\frac{\partial^{2} I_{H}^{2 \omega_{L}}}{\partial \Omega \partial \omega}\right)-\left(\frac{\partial^{2} I_{V}^{2 \omega_{L}}}{\partial \Omega \partial \omega}\right)\right) .
$$

Moreover, we note that for the $\mathrm{H}_{2}-\mathrm{Ar}$ mixture considered here the septor component of the $I_{H}$ CIHR spectrum computed in this study is stronger than its vector one. Significant values of the septor part of the hyperpolarizability tensor have been already reported. For instance, it was found experimentally that for $\mathrm{BaTiO}_{3}$ crystal the septor part of the SHG hyperpolarizability has the same magnitude as is vector part. ${ }^{50}$

In general, the CIHR spectra have two components separable by polarization effects. At this point an interesting general remark is appropriate. The above described horizontal $I_{H}$ geometry favors the septor part of the hyperpolarizability tensor. It is worth of notice that for these conditions the CIHR geometrical factors are equal to $8 / 105$ and $2 / 45$ for the vector part and the septor part, respectively. In this case the septor part geometrical coefficient is about 1.71 times higher than the vector one. So this geometry favors the septor part of the CI hyperpolarizability. For the polarized light scattering and the incident laser beam polarized perpendicularly to the scattering plane (the vertical geometry $-I_{V}$ ), the geometrical coefficients are equal to $2 / 9$ and $2 / 21$ for the vector part and the septor part, respectively. In this case the vector part geometrical coefficient is 2.33 times higher than the septor part geometrical one. So this geometry favors the vector

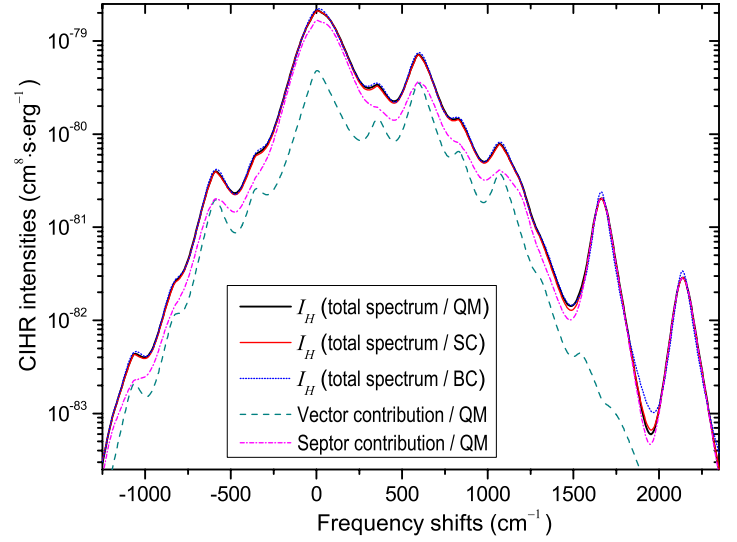

FIG. 3. (Color online) HR spectrum of $\mathrm{H}_{2}-\mathrm{Ar}$ in the horizontal $(H)$ polarization case for the wavelength of the YAG laser $\lambda_{L}=1064 \mathrm{~nm}$. The CIHR intensity (in absolute units: $\mathrm{cm}^{8} \mathrm{~s} / \mathrm{erg}$ ) is presented vs wavenumber (in $\mathrm{cm}^{-1}$ ) for three models: QM (thick solid line), SC (thin solid line), and BC (dot). However, the three curves are hardly distinguishable. From top to bottom at zero frequency: total intensity, septor contribution (dashed-dotted line), and vector contribution (dashed line).

part of the CI hyperpolarizability. This can be noticed on Fig. 4 , even if the septor contribution remains important at $\nu \approx 0 \mathrm{~cm}^{-1}$ and for high frequencies.

Successively, in Figs. 3 and 4 we compare the SC and QM results, finding quite decent agreement between the two methods of computing the CIHR spectra: the two lines can be barely distinguished (mind the logarithmic scale however) with relative discrepancies estimated between $\sim 5 \%$ and down to a fraction of percent for a relatively large range of the frequency shifts from 0.0 up to $1000.0 \mathrm{~cm}^{-1}$. Supposedly, the deviations may be accounted for by both physical and/or numerical factors. If the latter is the case, a more restrictive and accurate computing routine might result in even better agreement if necessary. On the other hand, for less massive systems (e.g., $\mathrm{H}_{2}-\mathrm{He}$ ) one can expect that quantal effect could play more predominant role in shaping the CIHR spectra leading to more noticeably divergent QM and SC profiles. Anyway, the results presented here can serve

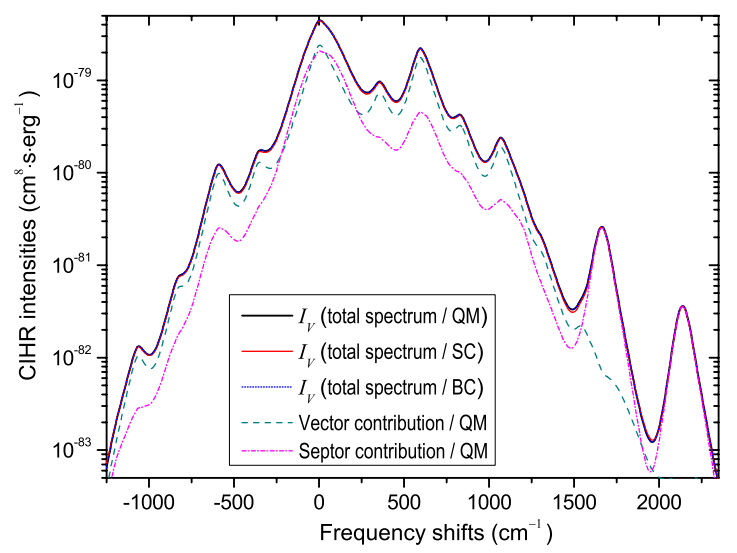

FIG. 4. (Color online) HR spectrum of $\mathrm{H}_{2}-\mathrm{Ar}$ in the vertical $(V)$ polarization case for the wavelength of the YAG laser $\lambda_{L}=1064 \mathrm{~nm}$. The CIHR intensity (in absolute units: $\mathrm{cm}^{8} \mathrm{~s} / \mathrm{erg}$ ) is presented vs wavenumber (in $\mathrm{cm}^{-1}$ ) for three models: QM (thick solid line), SC (thin solid line), and BC (dotted line). However, the three curves are hardly distinguishable. From top to bottom at zero frequency: total intensity, vector contribution (dashed line), and septor contribution (dashed-dotted line). 
as a solid proof of validity of both QM and SC formulas and numerical procedures. Eventually, it is necessary to mention here one more line plotted in Figs. 3 and 4 for the sake of comparison. It represents a spectral profile derived by means of the BC model. Again the difference between this graph and the QM and SC lines is rather small, yet the deviation calculated shows more significant divergence in this case.

Now, let us compare our binary $\mathrm{H}_{2}-\mathrm{Ar}$ CIHR intensities to the recently measured, monomer allowed HR spectra of small tetrahedral molecules. ${ }^{34}$ We chose the $\mathrm{CF}_{4}$ gas as a reference. The reduced monomer allowed integrated HR intensity (per one $\mathrm{CF}_{4}$ molecule) for the horizontal $(H)$ incident light polarization reads $S_{H}^{\mathrm{CF}_{4}}=16 / 35 b_{x y z}^{2}$. Taking into account that $b_{x y z}^{\mathrm{CF}_{4}}=5.4$ a.u., ${ }^{34}$ we obtain $S_{H}^{\mathrm{CF}_{4}}=1.019$ $\times 10^{-63} \mathrm{~cm}^{9} \mathrm{erg}^{-1}$. The respective reduced integrated CIHR intensity for $\mathrm{H}_{2}-\mathrm{Ar}$ pair is obtained using the values of the reduced zeroth moments ${ }^{(1 \lambda L)} M_{0}^{*}$ and ${ }^{(3 \lambda L)} M_{0}^{*}$ given in Table III. We have $\mathrm{S}_{H}^{\mathrm{H}_{2}-\mathrm{Ar}}=3.104 \times 10^{-67} \mathrm{~cm}^{9} \mathrm{erg}^{-1}$. Consequently for arbitrary density, the ratio of the $\mathrm{CIHR}_{2}-\mathrm{Ar}$ integrated intensity and the $\mathrm{CF}_{4}$ integrated $\mathrm{HR}$ intensity is $\rho_{\mathrm{H}_{2}} \rho_{\mathrm{Ar}} / \rho_{\mathrm{CF}_{4}} 3.05 \times 10^{-4}$, where $\rho_{i}$ denotes the density of the species $i$ in amagats.

\section{CONCLUSION}

In this work we have extended our previous numerical and theoretical treatment of nonlinear molecular properties (hyperpolarizabilies, to be exact) associated with nonlinear phenomena of molecular-radiation interaction (CIHR scattering). Within the framework of this study, calculations of the first hyperpolarizability tensor values have been performed on the grounds of QC methods. Subsequently these quantities, or rather their independent spherical components, were extracted and then, fitted to analytical formulas expressing their dependence on intermolecular distance, they were applied as the input data for analyses of the collisionally induced HR scattering in the systems composed of a linear molecule interacting with a noble gas atom. To the best of our knowledge, this is the first attempt to reach beyond the domain of previously considered CIHR effects in supermolecular systems of higher (linear) symmetry.

The theoretical and numerical approaches developed made it possible to formulate expressions and routines capable of yielding spectral CIHR profiles: translational, rotational, and their convolutions. The results obtained were discussed with regard to more detailed aspects of the spectra: the role of the vector and septor contributions, the share of the components associated with particular symmetry adopted hyperpolarizability contributions, the discrepancies between quantumlike and classicallike computational results, etc. As a result we have found out, for instance, the predominant role played by the septor spectral contribution, which feature is in contradiction with what one can observe in the previously discussed CIHR effects typical of supermolecular systems of linear symmetry. Another conclusion worth underlining here concerns the almost meaningless influence of the hyperpolarizability components of the order of 4-an important fact established within the theory which provides an evidence of a very rapid convergence of the series expansions determin- ing the hyperpolarizability properties and values. Last but not least, the good agreement between our quantum and SC approaches can be treated as a benchmark proof of both the method's feasibility and accuracy.

To sum up, we have created a suitable numerical and theoretical tool deriving and interpreting the CIHR spectra of light scattered in systems akin to $\mathrm{H}_{2}-\mathrm{Ar}$ that can be used in analyses of experimental data, which-due to incredible progress observed in molecular spectroscopy-should be soon at hand.

\section{ACKNOWLEDGMENTS}

This work has been supported, in part, by Grant No. 1P03B 08230 of the Polish Ministry of Education and Sciences.

We dedicate this paper to the memory of the late Professor Przemysław Szlachetka, our very good friend and an excellent physicist.

${ }^{1}$ B. J. Berne and R. Pecora, Dynamic Light Scattering (Wiley, New York, 1976).

${ }^{2}$ D. A. Long, Raman Spectroscopy (McGraw-Hill, New York, 1977).

${ }^{3}$ G. C. Tabisz, Molecular Spectroscopy (A Specialist Periodical Report), edited by R. F. Barrow, D. A. Long, and J. Sheridan (Chemical Society, London, 1979), Vol. 6, pp. 136-173.

${ }^{4}$ Phenomena Induced by Intermolecular Interactions, NATO ASI Series, edited by G. Birnbaum (Plenum, New York, 1985).

${ }^{5}$ Collision- and Interaction-Induced Spectroscopy, NATO Advanced Studies Institute, Series C: Mathematical and Physical Sciences Vol. 452, edited by G. C. Tabisz and M. N. Neuman (Kluwer Academic, Dordrecht, 1995).

${ }^{6}$ A. Elliasmine, J.-L. Godet, Y. Le Duff, and T. Bancewicz, Mol. Phys. 90, 147 (1997).

${ }^{7}$ A. Elliasmine, J.-L. Godet, Y. Le Duff, and T. Bancewicz, Phys. Rev. A 55, 4230 (1997).

${ }^{8}$ T. Bancewicz, A. Elliasmine, J.-L. Godet, and Y. Le Duff, J. Chem. Phys. 108, 8084 (1998).

${ }^{9}$ T. Bancewicz, K. Nowicka, J.-L. Godet, and Y. Le Duff, Phys. Rev. A 69, 062704 (2004).

${ }^{10}$ T. Bancewicz, Y. Le Duff, and J.-L. Godet, Advances in Chemical Physics Vol. 119, edited by M. Evans (Wiley, New York, 2001), p. 267.

${ }^{11}$ A. D. Buckingham, E. P. Concannon, and I. D. Hands, J. Phys. Chem. 98, 10455 (1994).

${ }^{12}$ X. Li, K. L. C. Hunt, J. Pipin, and D. M. Bishop, J. Chem. Phys. 105, 10954 (1996)

${ }^{13}$ T. Bancewicz, J.-L. Godet, and G. Maroulis, J. Chem. Phys. 115, 8547 (2001).

${ }^{14}$ W. Glaz and T. Bancewicz, J. Chem. Phys. 118, 6264 (2003).

${ }^{15}$ W. Glaz, T. Bancewicz, and J.-L. Godet, J. Chem. Phys. 122, 224323 (2005).

${ }^{16}$ W. Glaz, T. Bancewicz, J.-L. Godet, G. Maroulis, and A. Haskopoulos, Phys. Rev. A 73, 042708 (2006).

${ }^{17}$ T. Bancewicz, W. Głaz, and J.-L. Godet, J. Chem. Phys. 127, 134308 (2007).

${ }^{18}$ A. D. Buckingham, Adv. Chem. Phys. 12, 107 (1967).

${ }^{19}$ S. Kielich, Nonlinear Molecular Optics (PWN, Warsaw, 1977) (in Polish).

${ }^{20}$ L. Frommhold, Collision-Induced Absorption in Gases (Cambridge University Press, Cambridge, England, 1993).

${ }^{21}$ W. Meyer and L. Frommhold, Phys. Rev. A 34, 2936 (1986).

${ }^{22}$ J. M. St-Arnaud and T. K. Bose, J. Chem. Phys. 71, 4951 (1979).

${ }^{23}$ T. Bancewicz, V. Teboul, and Y. Le Duff, Mol. Phys. 81, 1353 (1994).

${ }^{24}$ B. M. Ladanyi and T. Keyes, Mol. Phys. 33, 1063 (1977).

${ }^{25}$ B. M. Ladanyi and T. Keyes, Mol. Phys. 33, 1247 (1977).

${ }^{26}$ A. D. Buckingham and G. C. Tabisz, Mol. Phys. 36, 583 (1978).

${ }^{27}$ L. Frommhold, Adv. Chem. Phys. 46, 1 (1981).

${ }^{28}$ N. Meinander, G. C. Tabisz, and M. Zoppi, J. Chem. Phys. 84, 3005 (1986). 
${ }^{29}$ N. Meinander, G. C. Tabisz, F. Barocchi, and M. Zoppi, Mol. Phys. 89, 521 (1996).

${ }^{30}$ F. Rachet, M. Chrysos, C. Guillot-Noël, and Y. L. Duff, Phys. Rev. Lett. 84, 2120 (2000).

${ }^{31}$ Optical, Electric and Magnetic Properties of Molecules: A Review of the Work of A. D. Buckingham, edited by D. C. Clary and B. J. Orr (Elsevier, Amsterdam, 1997).

${ }^{32}$ S. Kielich and T. Bancewicz, J. Raman Spectrosc. 21, 791 (1990).

${ }^{33}$ P. Kaatz and D. P. Shelton, Mol. Phys. 88, 683 (1996).

${ }^{34}$ R. D. Pyatt and D. P. Shelton, J. Chem. Phys. 114, 9938 (2001).

${ }^{35}$ P. D. Maker, Phys. Rev. A 1, 923 (1970).

${ }^{36}$ K. Clays and A. Persoons, Phys. Rev. Lett. 66, 2980 (1991).

${ }^{37}$ D. P. Shelton and J. E. Rice, Chem. Rev. (Washington, D.C.) 94, 3 (1994).

${ }^{38}$ J. Zyss and I. Ledoux, Chem. Rev. (Washington, D.C.) 94, 77 (1994).

${ }^{39}$ G. Maroulis and A. Haskopoulos, Chem. Phys. Lett. 358, 64 (2002).

${ }^{40}$ G. Maroulis, J. Phys. Chem. A 104, 4772 (2000).

${ }^{41}$ F. G. Fumi, Phys. Rev. A 86, 561 (1952).

${ }^{42}$ P. N. Butcher and D. Cotter, The Elements of Nonlinear Optics (Cambridge University Press, New York, 1990).

${ }^{43}$ X. Li, C. Ahuja, J. F. Harrison, and K. L. C. Hunt, J. Chem. Phys. 126, 214302 (2007).

${ }^{44}$ S. F. Boys and F. Bernardi, Mol. Phys. 19, 553 (1970).

${ }^{45}$ M. J. Frisch, G. W. Trucks, H. B. Schlegel et al., GAUSSIAN 98, Revision A.7, Gaussian, Inc., Pittsburgh, PA, 1998.

${ }^{46}$ T. Helgaker, P. Jørgensen, and J. Olsen, Molecular Electronic-Structure Theory (Wiley, Chichester, 2000).

${ }^{47}$ J. Stiehler and J. Hinze, J. Phys. B 28, 4055 (1995).
${ }^{48}$ G. Maroulis and D. M. Bishop, Chem. Phys. Lett. 128, 462 (1986).

${ }^{49}$ Z. Ożgo and S. Kielich, Physica B \& C 81, 151 (1976).

${ }^{50}$ J. Jerphagnon, D. Chemla, and R. Bonneville, Adv. Phys. 27, 609 (1978).

${ }^{51}$ T. Bancewicz, Computation in Modern Science and Engineering, American Institute of Physics Conference Proceedings 963 Vol. 1, Pt. A, edited by T. E. Simos and G. Maroulis (American Institute of Physics, New York, 2007).

${ }^{52}$ C. G. Gray and K. E. Gubbins, Theory of Molecular Fluids, Fundamentals Vol. 1 (Clarendon, Oxford, 1984).

${ }^{53}$ C. G. Gray and B. W. N. Lo, Chem. Phys. 14, 73 (1976).

${ }^{54}$ D. A. Varshalovich, A. N. Moskaliev, and V. K. Khersonskii, Quantum Theory of Angular Momentum (World Scientific, Singapure, 1988).

${ }^{55}$ T. Bancewicz, ICCMSE 2008, AIP Conference Proceedings (AIP, New York, in press).

${ }^{56} \mathrm{~T}$. Bancewicz and G. Maroulis, "Rotationally adapted studies of ab initio computed collision-induced hyperpolarizabilities: The case of $\mathrm{H}_{2}-\mathrm{Ar}$ pair," Phys. Rev. A (submitted).

${ }^{57}$ W. Głaz, J. Yang, J. D. Poll, and C. G. Gray, Chem. Phys. Lett. 218, 183 (1994).

${ }^{58}$ W. Głaz and G. C. Tabisz, Can. J. Phys. 79, 801 (2001).

${ }^{59}$ G. Birnbaum and E. R. Cohen, Can. J. Phys. 54, 593 (1976).

${ }^{60}$ N. Meinander, J. Chem. Phys. 99, 8654 (1993).

${ }^{61}$ R. J. LeRoy, J. S. Carley, and J. E. Grabenstetter, Faraday Discuss. Chem. Soc. 62, 169 (1976).

${ }^{62}$ H. L. Williams, K. Szalewicz, B. Jeziorski, R. Moszynski, and S. Rybak, J. Chem. Phys. 98, 1279 (1993).

${ }^{63}$ J. LeRoy and M. Hutson, J. Chem. Phys. 86, 837 (1987).

${ }^{64}$ E. A. Donley and D. P. Shelton, Chem. Phys. Lett. 215, 156 (1993). 